\title{
Neural Respiratory Drive Estimation in Respiratory sEMG with Cardiac Arrhythmias
}

\author{
Luis Estrada-Petrocelli, Senior Member, IEEE, Raimon Jané, Senior Member, IEEE and Abel Torres, \\ Senior Member, IEEE
}

\begin{abstract}
Neural respiratory drive as measured by the electromyography allows the study of the imbalance between the load on respiratory muscles and its capacity. Surface respiratory electromyography (sEMG) is a non-invasive tool used for indirectly assessment of NRD. It also provides a way to evaluate the level and pattern of respiratory muscle activation. The prevalence of electrocardiographic activity (ECG) in respiratory sEMG signals hinders its proper evaluation. Moreover, the occurrence of abnormal heartbeats or cardiac arrhythmias in respiratory sEMG measures can make even more challenging the NRD estimation. Respiratory SEMG can be evaluated using the fixed sample entropy (fSampEn), a technique which is less affected by cardiac artefacts. The aim of this work was to investigate the performance of the fSampEn, the root mean square (RMS) and the average rectified value (ARV) on respiratory SEMG signals with supraventricular arrhythmias (SVA) for NRD estimation. fSampEn, ARV and RMS parameters increased as the inspiratory load increased during the test. fSampEn was less influenced by ECG with SVAs for the NRD estimation showing a greater response to respiratory sEMG, reflected with a higher percentage increase with increasing load (228\% total increase, compared to $142 \%$ and $135 \%$ for $\mathrm{ARV}$ and RMS, respectively).
\end{abstract}

Clinical Relevance-Improvement of the NRD estimation in patients with cardiac arrhythmias contributes to a better assessment of respiratory muscle activity in patients with chronic respiratory diseases and cardiovascular comorbidities.

\section{INTRODUCTION}

Electromyography is a reliable and widely used tool for monitoring and evaluating the muscles involved in the respiration. Electromyography can be measured using invasive methods, such the use of an multipair oesophageal electrode catheter positioned at the level of the crural portion of the diaphragm. Although it is possible to obtain direct information about the respiratory muscle activity with this technique, its use requires a trained clinical staff. Alternatively, another approach that has gained widespread acceptance in the last few years is the non-invasive recording of respiratory electromyographic signal (sEMG), using surface electrodes placed on the muscle of interest to be measured. Because of their simplicity and ease of use, non-invasive acquisitions are an attractive alternative for the study of respiratory muscle activity both inside and outside a hospital environment [1].

This work was supported in part by the CERCA Program/Generalitat de Catalunya, in part by the Secretaria d'Universitats i Recerca de la Generalitat de Catalunya under grant GRC 2017 SGR 01770 and in part by the spanish grant RTI2018-098472-B-I00 (MCIU/AEI/FEDER, UE) and the Biomedical Research Networking Centre in Bioengineering, Biomaterials and Nanomedicine (CIBER-BBN). L. Estrada-Petrocelli, A. Torres and R. Jané are with the Institut de Bioenginyeria de Catalunya (IBEC), the Barcelona
Importantly, respiratory electromyographic recordings are contaminated by bioelectric signals, particularly from cardiac activity (ECG) thus limiting their clinical interpretation. The influence of ECG on respiratory sEMG signals may vary depending on the type of electrodes and configuration, as well as the recording site, altering the shape and intensity of both signals [2], [3]. Reduction of cardiac interference in respiratory sEMG by applying processing techniques is an important step for its further analysis as outlined in [4]. This issue is relevant for indirectly quantify the neural respiratory drive (NRD) in healthy and chronic obstructive pulmonary disease (COPD) patient populations using electromyographic techniques. NRD as measured by electromyography allows the study of the imbalance between the load on the respiratory muscles and its capacity, contributing to assess the level and pattern of respiratory muscle activation and making it possible to differentiate between healthy subjects and COPD patients [5] with comorbidities [6]. In addition to the normal ECG contamination on respiratory sEMG signals, the occurrence of abnormal heartbeats or cardiac arrhythmias can hamper the NRD estimation, particularly in COPDs where the presence of supraventricular arrhythmias are common [11], thus making even more challenging its evaluation. The fixed sample entropy (fSampEn) is a technique for the estimation of amplitude changes in myographic signals [8]. It has demonstrated the ability to reduce the cardiac interference described as an repetitive- and impulsive-like noise [8]. However, fSampEn has not been evaluated in case of respiratory sEMG with arrhythmias. The aim of the current study was to assess the performance of fSampEn on respiratory sEMG signals in presence of cardiac arrhythmias. To this end, the influence of two supraventricular arrhythmias were investigated: supraventricular tachycardia and atrial flutter.

\section{MATERIAL AND METHODS}

\section{A. Study Population}

Five healthy non-smoking volunteers (all males), age: 30.0 (31.0-32.5) years, height: $1.75(1.71-1.81) \mathrm{m}$, weight: 82.0 $(75.8-85.5) \mathrm{kg}$ and body mass index $\left.25.9(23.8-27.78) \mathrm{kg} / \mathrm{m}^{2}\right)$ with no history of cardiac, respiratory or neuromuscular disease were enrolled in the study. The experimental data for this work were collected in a previous study, which was approved by the Institutional Review Board (IRB) of the

Institute of Science and Technology (BIST), Universitat Politècnica de Catalunya (UPC)-BarcelonaTech, Campus Diagonal-Besòs, Av. d'Eduard Maristany, 10-14, building C, 5th floor, 08930, Barcelona, Spain and with the CIBER-BBN (e-mails: \{lestrada, atorres rjane\} @ibecbarcelona.eu). A. Torres and R. Jané are also with the UPC·BarcelonaTech. L. EstradaPetrocelli is also with the Facultad de Ingeniería, Universidad Latina de Panamá, Apartado Postal 0823-00933, Panama City, Republic of Panama. 

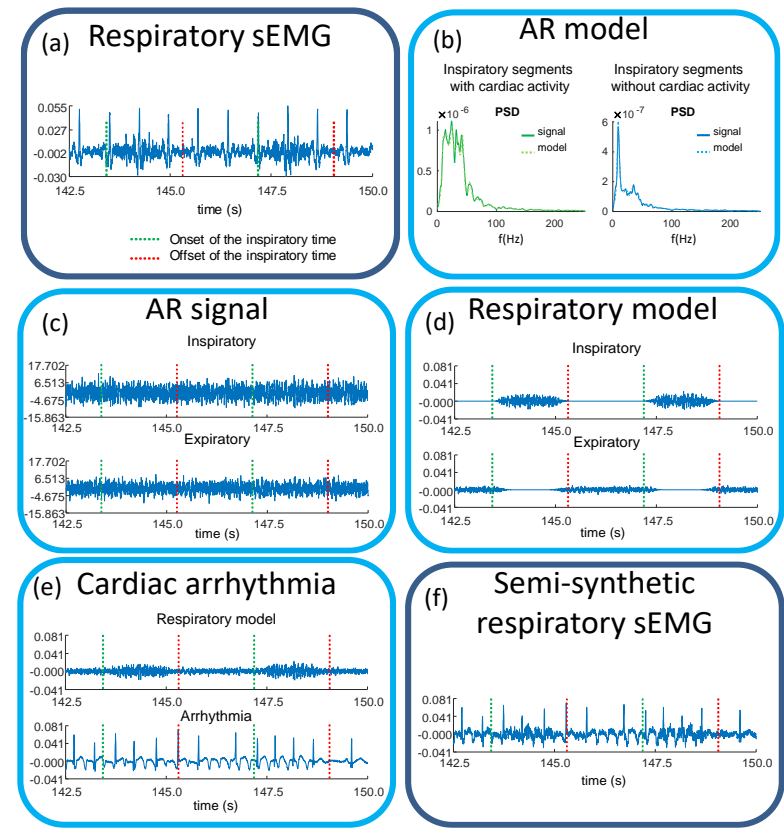

(f)

(f) Semi-synthetic respiratory sEMG

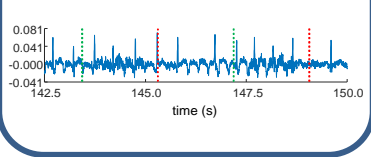

Figure 1. Block diagram for semi-synthetic respiratory sEMG signal generation. (a) Recorded respiratory sEMG, (b) inspiratory and expiratory power spectral density (PSD) for the respiratory sEMG without ECG and PSD of their corresponding autoregressive (AR) model, (c) semi-synthetic inspiratory and expiratory sEMG without ECG from their AR model, (d) semi-synthetic inspiratory and expiratory sEMG signals multiplied by their corresponding inspiratory and expiratory envelopes, (e) semi-synthetic respiratory sEMG without ECG and cardiac arrhythmia, and (f) semi-synthetic respiratory sEMG with cardiac arrhythmia.

Institute for Bioengineering of Catalonia, Barcelona, Spain (IRB_IBEC_RJANE_2016_01) [8]. Signed, written informed consent was obtained from all participants prior to their participation in the study.

\section{B. Experimental Setup}

Respiratory sEMG was obtained using a pair of adhesive disposable disc electrodes (Ag- $\mathrm{AgCl}, 11-\mathrm{mm}$ diameter, pregelled, foam electrode 50/PK - EL501, BIOPAC Systems, Santa Barbara, CA, USA) in bipolar configuration placed close to the apposition zone of the costal portion of the diaphragm on the lower right chest wall. Surface electrodes were attached on the skin surface, over the $7^{\text {th }}$ and $8^{\text {th }}$ intercostal space, one below the other on the anterior axillary line and the midclavicular line above the costal margin [8]. A common reference electrode was placed on the right ankle. The skin of the participants was appropriately prepared prior to electrode application with an abrasive gel (Nuprep, Weaver and Company, Aurora, CO, USA) and cleaned with alcohol. Respiratory sEMG signals were conditioned using a modular differential amplifier (EMG100C, BIOPAC Systems, Inc.) with an analogue band-pass filter using cut-off frequencies between $1 \mathrm{~Hz}$ and $500 \mathrm{~Hz}$ and a gain of 1000 . Inspiratory mouth pressure (Pmouth) was measured with a differential pressure transducer (TSD160, BIOPAC Systems, Inc.) plugged into to a modular differential amplifier (DAC100C, BIOPAC Systems Inc.) with an analogue low-pass filter using a cut-off frequency of $300 \mathrm{~Hz}$ and a gain of 50 . All signals were digitized at a sampling rate of $2000 \mathrm{~Hz}$ using a 16-bit analog-to-digital converter data acquisition system (MP150,
BIOPAC Systems Inc.) and stored on a computer via the accompanied software (AcqKnowledge software v.3.2 BIOPAC Systems Inc.).

\section{Respiratory tests}

Participants performed an inspiratory threshold loading test (ILT) in which they firstly breathed at quiet tidal breathing and then the inspiratory load on the respiratory system was increased using a hand-held inspiratory muscle training device (Threshold IMT, Phillips Respironics, Amsterdam, The Netherlands). Four inspiratory loads at 9, 17, 25 and $33 \mathrm{~cm}$ $\mathrm{H}_{2} \mathrm{O}$ were imposed. ILT lasted $1 \mathrm{~min}$ for each increase in inspiratory load with $2 \mathrm{~min}$ of rest. Participants were asked to seat on a chair, in a straight posture with their arms by their sides while wearing a nose clip to prevent air leakage through the nostrils.

\section{ECG signals with Cardiac Arrhythmias}

ECG with supraventricular arrhythmias (SVA) were obtained from the publicly available MIT-BIH Arrhythmia Database [9]. Two SVA of $1 \mathrm{~min}$ each were evaluated, according to annotated rhythms: supraventricular tachyarrhythmias (SVTA) and atrial flutter (AFL). ECG signals were obtained from the modified limb lead II (MLII) sampled at $256 \mathrm{~Hz}$ with 11-bit resolution over a range of 10 $\mathrm{mV}$. The MLII lead was collected using surface electrodes placed on the torso for monitoring long-term heart activity.

\section{Semi-synthetic sEMG Signals}

A set of semi-synthetic respiratory sEMG signals were generated from each of the participants' respiratory sEMG signals. The steps for generating a respiratory synthetic signal were developed according to our previous study [8]. First, for a given respiratory sEMG signal, the inspiratory and expiratory power spectral density (PSD) for the respiratory s EMG without ECG noise were estimated. Then, the inspiratory and expiratory PSD of their corresponding autoregressive (AR) models were calculated. Next, semisynthetic inspiratory and expiratory sEMG signals without ECG noise from their AR models were obtained. After that, respiratory cycles were derived by multiplying the inspiratory and expiratory sEMG AR models each lasting $1 \mathrm{~min}$, by inspiratory and expiratory envelopes (with a frequency of 16 bpm). Finally, a semi-synthetic respiratory sEMG signal was obtained by adding the semi-synthetic inspiratory and expiratory sEMG signals and the cardiac arrhythmias (Fig. 1).

\section{Signal Processing}

Acquired respiratory sEMG signals were digitally bandpass filtered using a zero-phase fourth-order Butterworth filter with cut-off frequencies of 5 and $400 \mathrm{~Hz}$. Pmouth was lowpass filtered using a zero-phase fourth-order Butterworth filter with a cut-off frequency of $5 \mathrm{~Hz}$. ECG with SVAs were highpass filtered using a zero-phase fourth-order Butterworth filter with a cut-off frequency of $1 \mathrm{~Hz}$ and resampled to $2000 \mathrm{~Hz}$. Respiratory sEMG signals were analyzed using the root mean square (RMS) and the average rectified value (ARV). In addition, the fixed sample entropy (fSampEn), a robust method to the presence of ECG that permits the evaluation of respiratory myographic signals was utilized [8]. Calculation of fSampEn requires the setting of three input parameters: the embedded dimension $(m)$ related to the length of patterns to be compared, the tolerance value $(r)$ representing the threshold 
(a)

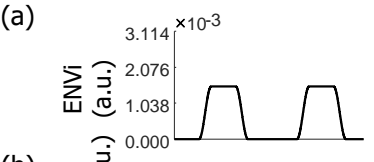

(b)

(c)

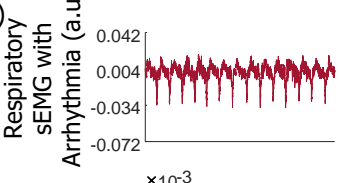

$$
\text { 产 }
$$

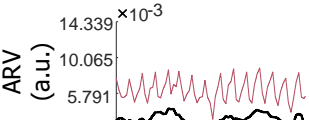

(d)

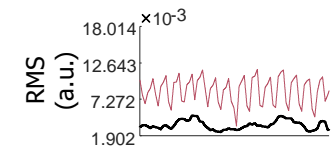

(e)

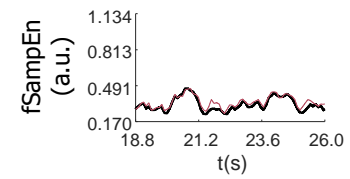

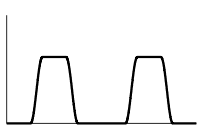
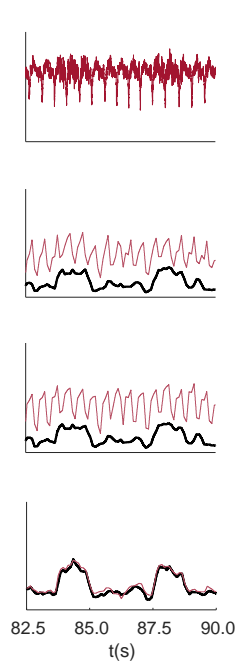
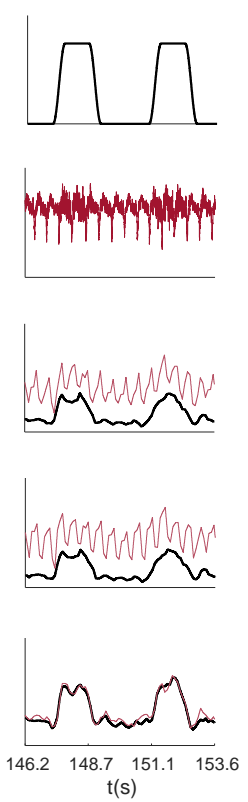
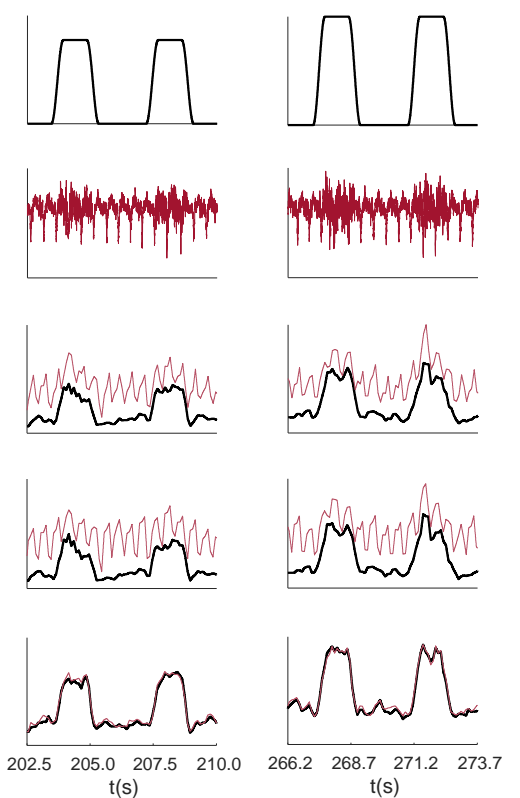

Figure 2. Evaluation of semi-synthetic respiratory sEMG signals with supraventricular tachyarrhythmias. (a) Inspiratory envelope (ENVi), (b) semisynthetic respiratory sEMG, evaluation of (c) ARV, (d) RMS and (e) fSampEn without (black) and with (red) cardiac arrhythmias. Two representative breathing cycles are shown at five levels of ENVi corresponding to quiet breathing and inspiratory loads of $9,17,25$ and $33 \mathrm{~cm} \mathrm{H}_{2} \mathrm{O}$.

within which the similarity between two patterns is compared and the window size $(N)$ representing the length of the timeseries to be evaluated. The $m$ input parameter was set equal to 1 , the $r$ parameter was set to 0.3 time the standard deviation of the whole respiratory sEMG signal whilst $N$ was set equal to $300 \mathrm{~ms}$ [10]. RMS, ARV and fSampEn were calculated on semi-synthetic respiratory sEMG signals using a moving of window of analysis with $90 \%$ overlapping.

\section{E. Data Analysis}

The peak value for RMS, ARV and fSampEn within each inspiratory cycle was detected for e ach level of inspiratory load. Data were normalized by the average value to the lowest inspiratory load and expressed as percentages. Signals were processed and analyzed using custom-developed software on MATLAB (v. R2019b, Natick, MA, USA).

\section{RESULTS}

Figs. 2 and 3 show an example of the inspiratory envelope and the performance of the ARV, RMS and fSampEn on semi- (a)

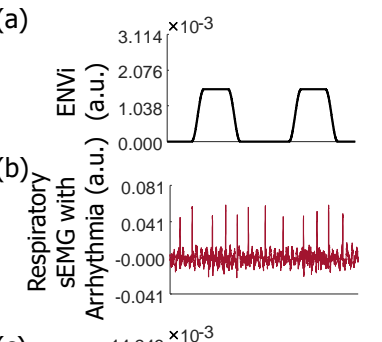

(c)

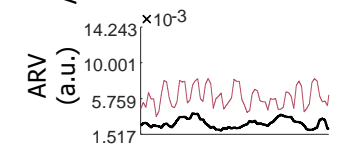

(d)

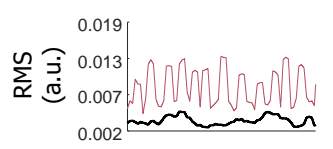

(e)
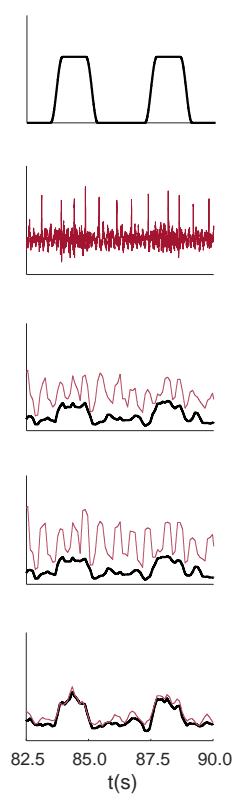
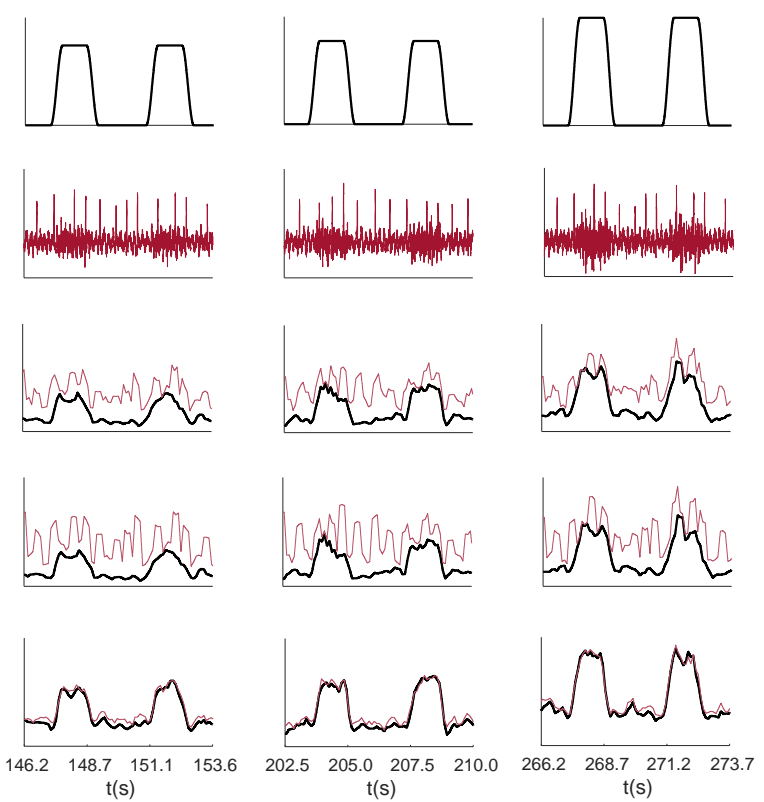

Figure 3. Evaluation of semi-synthetic respiratory sEMG signals with atrial flutter. (a) Inspiratory envelope (ENVi), (b) semi-synthetic respiratory sEMG, evaluation of (c) ARV, (d) RMS and (e) fSampEn without (black) and with (red) cardiac arrhythmias. Two representative breathing cycles are shown at five levels of ENVi corresponding to quiet breathing and inspiratory loads of 9, 17,25 and $33 \mathrm{~cm}_{2} \mathrm{O}$. 

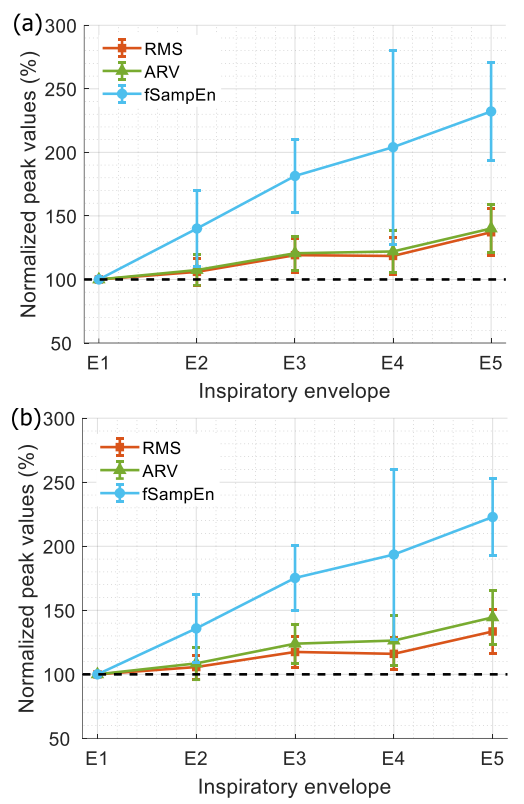

Figure 4. Evaluation of neural respiratory drive in semi-synthetic respiratory sEMG signals with (a) supraventricular tachyarrhythmias and (b) atrial flutter using ARV, RMS and fSampEn calculated at different levels of amplitude of synthetic inspiratory envelopes and normalized to the first mean value. E1 to E5 represent the synthetic inspiratory envelopes corresponding to quiet breathing and inspiratory loads of $9,17,25$ and $33 \mathrm{~cm} \mathrm{H}_{2} \mathrm{O}$.

synthetic respiratory sEMG signals without and with arrhythmias. In Fig. 2, semi-synthetic respiratory sEMG was combined with SVTA, whose characteristic is an abnormally fast heartbeat. It can be observed that the influence of SVTA is more prominent in ARV and RMS than in fSampEn because the fast heart rhythm clusters the ECG waves and thus making difficult to evaluate the electromyographic content.

In Fig. 3, semi-synthetic respiratory sEMG was combined with AFL, characterized by an abnormal high rate of the atria. Consequently, ARV and RMS are more sensitive not only to the occurrence of the QRS complex but also to the repetitive pattern of $\mathrm{P}$ waves generated during AFL. In both cases, the higher the respiratory activity (represented as inspiratory envelopes), the higher the respiratory sEMG and thus the lower influence of cardiac arrhythmias. The breathing cycles were more defined when using the fSampEn than with classical amplitude estimators.

Fig. 4 depicts the maximum values achieved during inspiration as the load increased. For each parameter, data was normalized to the mean value corresponding to quiet breathing. On the last inspiratory load, the mean value of the fSampEn percentage increases by $228 \%$, compared to $142 \%$ and $135 \%$ of the ARV and RMS, respectively.

\section{DISCUSSION AND CONCLUSIONS}

Estimation of amplitude variations in respiratory sEMG signals is an important issue for the NRD estimation. The challenge faced when analyzing these respiratory signals is to reduce the heart interference. In addition, the occurrence of cardiac arrhythmias can complicate the analysis. Current techniques used for processing the respiratory sEMG signal are focused on the reduction of ECG and only a few take into consideration the presence of arrhythmias [7]. Alternatively, ectopic beats can be isolated and removed from the analysis of respiratory signals [7]. Although this is a straightforward way to analyze the electromyographic content, certain studies may require the evaluation of entire signals, especially if a patient is unable to perform a respiratory test for a long time. The main finding of the work is that the use of the fSampEn can be employed for the evaluation of respiratory sEMG signals with arrhythmias of supraventricular origin. fSampEn was the least influenced parameter by ECG with SVAs, showing a greater response to respiratory sEMG, reflected with a higher percentage increase with increasing load, compared to ARV and RMS (Fig. 4). fSampEn enhanced the electromyographic information related to the muscle respiratory activation during the ILT. These results are in keeping with that proposed in [8], in which synthetic and real respiratory sEMG signals were investigated with fSampEn. Potential studies can be carried out in COPD patients with comorbidities associated to cardiovascular disease with prevalent ECG with SVA [11].

This preliminary study has demonstrated the remarkable performance of using fSampEn for the respiratory sEMG evaluation in presence of ECG with SVA. However, the limitation of this study is that respiratory sEMG signals assessed are semi-synthetics in nature. Future studies would require evaluating the fSampEn in recorded respiratory sEMG signals with SVAs in respiratory chronic patients for the improvement of the NRD evaluation.

\section{REFERENCES}

[1] M. Ràfols-de-Urquía, L. Estrada, J. Estévez-Piorno, L. Sarlabous, R. Jané, and A. Torres, "Evaluation of a wearable device to determine cardiorespiratory parameters from surface diaphragm electromyography," IEEE J. Biomed. Heal. Informatics, vol. 23, no. 5, pp. 1964-1971, Sep. 2019.

[2] I. M. M. Dos Reis, D. G. Ohara, L. B. Januário, R. P. Basso-Vanelli, A. B. Oliveira, and M. Jamami, "Surface electromyography in inspiratory muscles in adults and elderly individuals: A systematic review," J. Electromyogr. Kinesiol., vol. 44, pp. 139-155, Feb. 2019.

[3] M. Puurtinen, J. Viik, and J. Hyttinen, "Best electrode locations for a small bipolar ECG device: Signal strength analysis of clinical data," Ann. Biomed. Eng., vol. 37, no. 2, pp. 331-336, 2009.

[4] R. W. van Leuteren, G. J. Hutten, C. G. de Waal, P. Dixon, A. H. van Kaam, and F. H. de Jongh, "Processing transcutaneous electromyography measurements of respiratory muscles, a review of analysis techniques," J. Electromyogr. Kinesiol., vol. 48, pp. 176-186, Oct. 2019.

[5] C. J. Jolley et al., "Neural respiratory drive in healthy subjects and in COPD," Eur. Respir. J., vol. 33, no. 2, pp. 289-297, Feb. 2009.

[6] L. Estrada-Petrocelli et al., "The relationship between the diaphragm mechanomyogram and breathlessness during inspiratory loading in chronic obstructive pulmonary disease," Eur. Respir. J., vol. 54, no. suppl 63, p. OA481, Sep. 2019.

[7] C. Maier and H. Dickhaus, "Extraction of respiratory myogram interference from the ECG and its application to characterize sleeprelated breathing disorders in atrial fibrillation," J. Electrocardiol., vol. 47, no. 6, pp. 826-830, 2014.

[8] L. Estrada, A. Torres, L. Sarlabous, and R. Jané, "Improvement in neural respiratory drive estimation from diaphragm electromyographic signals using fixed sample entropy," IEEE J. Biomed. Heal. Informatics, vol. 20, no. 2, pp. 476-485, Mar. 2016.

[9] G. B. Moody and R. G. Mark, "The impact of the MIT-BIH arrhythmia database," IEEE Eng. Med. Biol. Mag., vol. 20, no. 3, pp. 45-50, May 2001.

[10] L. Estrada, A. Torres, L. Sarlabous, and R. Jané, "Influence of parameter selection in fixed sample entropy of surface diaphragm electromyography for estimating respiratory activity," Entropy, vol. 19, no. 9, p. 460, Sep. 2017.

[11] S. P. Bhatt and M. T. Dransfield, "Chronic obstructive pulmonary disease and cardiovascular disease," Transl. Res., vol. 162, no. 4, pp. 237-251, Oct. 2013. 